\title{
Ultrasonic thermometry by a localized measurement of the longitudinal wave velocity using sound field information
}

This paper was downloaded from TechRxiv (https://www.techrxiv.org).

\section{LICENSE}

CC BY 4.0

SUBMISSION DATE / POSTED DATE

$01-11-2021 / 05-11-2021$

\section{CITATION}

Leipner, Emanuel; Wolf, Mario; Kühnicke, Elfgard; Kupsch, Christian (2021): Ultrasonic thermometry by a localized measurement of the longitudinal wave velocity using sound field information. TechRxiv. Preprint. https://doi.org/10.36227/techrxiv.16912177.v1

$\mathrm{DOI}$ 


\title{
Ultrasonic thermometry by a localized measurement of the longitudinal wave velocity using sound field information
}

\author{
Mario Wolf, Emanuel Leipner, Christian Kupsch, Member, IEEE, Elfgard Kühnicke
}

\begin{abstract}
Temperature monitoring during medical interventions such as hyperthermia or radio frequency ablation is a demanding task. An alternative to expensive MRI measurements are ultrasound measurements exploiting the dependence of sound velocity on temperature. In this paper, a new measurement technique is presented that determines the sound velocity spatially resolved in tissue phantoms with the aim of temperature monitoring. The measurements are performed with annular arrays operating element-wise in pulse-echo mode to allow synthetic focusing. Unlike speckle techniques, which also evaluate echoes from scatterers, this method allows to determine the true position of scatterers and absolute values of local sound velocity, rather than evaluating only relative changes. This is achieved by varying the set of delay times used for focusing and determining the set that gives the maximum amplitude of the focused echo. The measurement technique is described in detail and measurement results are shown. The achievable resolution $(1 \mathrm{~mm}$ axial and $0.4 \mathrm{~mm}$ lateral) and accuracy of $98 \%$ are determined. Sound field simulations are used to model the measurement process and vary measurement parameters. Thus, the effect of parameter variations on the achievable accuracy can be evaluated very precisely.
\end{abstract}

Index Terms-ultrasound, temperature monitoring, sound velocity measurement, scattering, sound-field simulation, annular arrays.

\section{INTRODUCTION}

C ONVENTIONAL ultrasonic thermometry, known as "thermal strain imaging" or "temporal strain imaging," exploits the fact that the speed of sound (SoS) of the longitudinal wave is dependent on the temperature of the examined tissue. These approaches are based on an echo shift. Originally, the echo shift was determined along a segment of the RF signal (1D) inside a correlation window[1], [2],[3]. The now widely used speckle tracking is a refined version of this original approach, using a 2-D complex cross-correlation of two subsequent frames of the RF echo [4][5] [6]. For both of these shift approaches, the spatial resolution depends on signal processing parameters, such as bandwidth, cross-correlation, window size, and window overlap. The size of the window (the spatial kernel for speckle tracking respectively) is chosen in order to achieve a trade-off between signal to noise ratio (SNR) and spatial resolution [3], [5] and takes into consideration both the echogenicity and the examination depth.

M. Wolf, E. Leipner and E. Kühnicke are with the solid State electronics laboratories, Technical University of Dresden, Germany, e-mail: mario.wolf@tu-dresden.de.

C. Kupsch is with the Measurement, Sensor and Embedded Systems Laboratory (MSE Lab) at TU Bergakademie Freiberg, Germany.
Even though shift approaches are the established approach in thermometry, they come with significant limitations. First, all echo shift methods estimate relativ sound velocity changes but are not capable of deternining an absolute sound velocity. Therefore, second, an initial velocity needs to be assumed. Third, the shift of the echo results from a deviation of the local SoS and from a tissue expansion, both caused by the altered temperature. Liu et al. assume that expansion is negligible [6]. In contrast, Arnal et al. observe that part of a lesion expands and another one shrinks simultaneously [7], while Sou et al. state that the effects of expansion and SoS-change could cancel each other out [8]. Despite all the insufficiencies listed above, recent research aims to refine the shift approach while neglecting potential tissue expansions [9], [10], [11], [12].

Furthermore, only limited data for the actual relationship between temperature $(T)$ and SoS $(c)$ are available. Even water exhibits a high complexity: The temperature-dependencecurve of SoS does not increase monotonically with temperature. Only for low temperatures, the curve can be approximated by a linear monotonic relationship, while it flattens for higher temperatures and reaches its maximum around $75^{\circ} \mathrm{C}$. Moreover, the SoS curve varies for different types of tissues as well as between individuals. It is also highly dependent on fat content, as shown for liver samples in [13]. According to modeling by Miller et al., the curve for a normal human liver resembles the one of water yet with a maximum at $60^{\circ} \mathrm{C}$. For a liver with an intermediate fat content, the maximum is predicted to appear at $45^{\circ} \mathrm{C}$, while a marked fatty liver peaks at $35^{\circ} \mathrm{C}$. For temperatures beyond the peak, no one-to-one relationship exists.

Therefore, conventional ultrasonic thermometry causes major uncertainties at temperatures above $50{ }^{\circ} \mathrm{C}$ and is generally only used for hyperthermia monitoring [11], [10]. Monitoring of High Intensity Focusssed Ultrasound treatment with Twaves [14] [15] omit temperature determination and focus on the detection and size of irreversible tissue damage. Shear wave elastography (SWE) is widely implemented in modern medical devices yet the shear wave velocity estimation depends on the respective algorithm [16], [17], [18], [19] and on the geometry of the sound beam and the parameters of the medium. Therefore, SWE is not feasible for quantitative statements [16]. The generated shear wave amplitude is evaluated with speckle tracking, which leads to the disadvantages of the shift methods described above. In [20] "time reversal" algorithms reduce the decorrelation of the shear wave signal, but the proposed algorithm only works if the source position 
is known and the tissue expansion due to heating is negligible. In [21], [22], [23], [24], [25], it is stated that a main source of error is the uncertainty of the tracking position. Corrections are attempted with a-priori information [25] or calibration of the tracking position [24].

Recent research explores the potential of SoS maps for cancer imaging [26], transcranial ultrasound imaging [27] and adaptive beamforming [28]. Numerous wave-based approaches have been developed [29][26][30][31][32] [33]. So far these wave-based approaches achieve a very accurate speed of sound through averaging over large areas. They have not yet yielded highly localized speed maps or time resolved resolutions, which are both necessary for a successful thermometry application.

Building on [34],[35], we report a novel wave-based approach that provides SoS measurements with high spatial and temporal resolution. In this paper we demonstrate the accuracy of this novel approach. in contrast to conventional ultrasonic imaging, temperature monitoring, and shear wave elastography, which commonly use arrays with 128 or 264 line elements, we apply a pre-focused sparse annular area with $N=6$ elements (one central element and 5 rings). One major advantage of annular arrays is the fact that they can generate the necessary focus spot within the range of the wavelength with a significantly reduced number of elements. In section 2, we explain the effect of different focusing regimes by means of sound field simulations for the used annular array and demonstrate the adequacy of the principle idea of the novel approach. Subsequently, we describe, in section 3, the measurement setup as well as the general measurement procedure. In 4, we show the accuracy of the method on the basis of experimental results and present sound velocity measurements in a tissue phantom with a temperature gradient. In 5, we predict, by means of modeling, the achievable level of accuracy in order to fit the array for the particular application.

\section{THEORETICAL FOUNDATIONS}

\section{A. Sound Field Simulations for the Annular Array}

A detailed knowledge of the sound field is necessary to study the effects described in this section. Further they are used to validate the proposed new approach and to estimate its measurement capabilities.

The sound-field simulations are based on harmonic Green's functions in combination with a point source synthesis [36]. For annular arrays, the calculations are done separately for each ring element. The area is discretized and approximated with uniformly distributed point sources. While the Green's functions factor in the parameters of the propagation medium as well as the emitted center frequency, the point source synthesis accounts for the shape and size of the annular array elements. For sound fields radiated from finitely extended sources, the fields of all point sources are superimposed [37],[38] . This method is applied for each ring element to simulate the field generated in the medium. We provide the fields of the used array in Figure 1 for a medium with $c_{\text {med }}=1500 \mathrm{~m} / \mathrm{s}$ at $f=10 \mathrm{MHz}$. The shown fields represent the resulting pressure distributions $P_{i}(r, z, f)$ for the harmonic excitation of the elements.

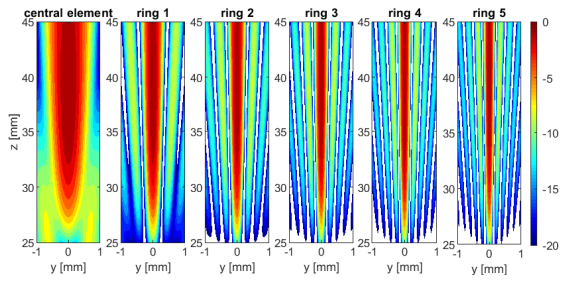

Fig. 1. Calculated sound fields of the six elements of an annular array for a medium with $c=1500 \mathrm{~m} / \mathrm{s}$. Find die array geometry in Table I

In general, measurements are done in pulse echo mode with short signals of limited bandwidth. To calculate the resulting transient pressure signal at any observation point, the signal is generated by a harmonic synthesis. That means that the sound field is calculated for several frequencies. The frequencies are determined by the spectrum $S_{0}(f)$ of the excitation signal $s_{0}(t)$. Afterwards the spectral components of the sound field are multiplied with the spectral components of the excitation signal and an inverse Fourier transform is applied. The received signal on element $m$ for a point reflector at the position $(r, z)$ for emission with element $n$ is calculated via: $s_{i j}(r, z, t)=$ ifft $\left(S_{0}(f) P_{n}(r, z, f) P_{m}(r, z, f)\right)$. In[39], we have shown that the harmonic field at centre frequency reliably predicts the location of the maximum and sufficiently approximates the maximal amplitude of the resulting signal. Therefore, the subsequent discussion relies exclusively on harmonic sound field calculations. This means that the harmonic sound field calculations give the emittet pressure distribution in a medium on the one hand but that they are also a measure for the amplitude of the signal reflected by a point-scatterer one the other hand.

\section{B. Focussing}

Focussing means that all wave parts interfere constructively at a assigned focus point. This can be realized by a manipulation of the propagation path, using a lens for single element transducers, or by delaying the signal parts from the elements of an array, also known as beamforming. In our approach, we rely on a focussing regime $F\left(z_{\mathrm{foc}}, c_{\mathrm{foc}}\right)$ that is set in order to produce a sensitive focus area at the distance $z_{\mathrm{foc}}$ in a medium with a velocity $c_{\text {foc }}$. In order to determine the appropriate delay times, we initially define for each element $n$ of the annular array: $S_{n}\left(r_{e l, n}, z_{e l, n}\right)$ is the point from which the wave is geometrically emitted. The time of fight $(T o F) t_{n}$ from each element $n$ to the assigned depth $z_{\text {foc }}$ is calculated by:

$$
t_{n}=\frac{\sqrt{\left(z_{\mathrm{foc}}-z_{e l, n}\right)^{2}+r_{e l, n}^{2}}}{c_{\mathrm{foc}}}
$$

The focussing of the measured signals is realized by delaying and superimposing the signals $s_{n m}$ of all combinations of emmiters $n$ and receivers $m$ :

$$
s_{\text {foc }}(t)=\sum_{n=1}^{N} \sum_{m=1}^{N} s_{n m}\left(t-t_{n}-t_{m}+2 t_{1}\right)
$$




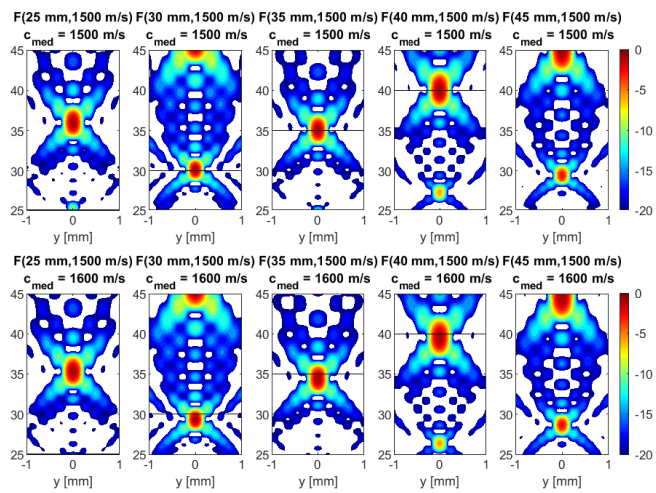

Fig. 2. Resulting focussed sound fields in media with $S o S=1500 \mathrm{~m} / \mathrm{s}$ (upper row) and $S o S=1600 \mathrm{~m} / \mathrm{s}$ (lower row) in various depth. The same sound velocity $c_{\text {foc }}=1500 \mathrm{~m} / \mathrm{s}$ is used for focussing for both media. Note that focussing at $25 \mathrm{~mm}$ is not possible, as this is in the nearfield of the single elements (see Figure 1). Focussing is possible in a range of $30 \mathrm{~mm}$ to $55 \mathrm{~mm}$ with this array. There are notable side structures in the sound field, e.g. at $28 \mathrm{~mm}$ for focussing on $40 \mathrm{~mm}$. They must be considered when evaluating measured data, but are generally not a problem. In the upper row the focus is located at the assigned position ( $30 \mathrm{~mm}, 35 \mathrm{~mm}, 40 \mathrm{~mm}$ and $45 \mathrm{~mm}$ ), for the lower row we obtain it at $29.32 \mathrm{~mm}, 34.38 \mathrm{~mm}, 38.48 \mathrm{~mm}$ and $44.63 \mathrm{~mm}$

For the harmonic sound field of the array, the focusing regime is realized by phase shifting and superimposing the element fields $P_{n}$.

$$
\begin{gathered}
P_{\text {foc }}=\sum_{n=1}^{N} \sum_{n=1}^{N} P_{n} P_{m} e^{-i 2 \pi f\left(t_{n}+t_{m}-2 t_{1}\right)} \\
P_{\text {foc }}=\left(\sum_{n=1}^{N} P_{n} e^{-i 2 \pi f\left(t_{n}-t_{1}\right)}\right)^{2}
\end{gathered}
$$

The focussing regime $F\left(z_{\mathrm{foc}}, c_{\mathrm{foc}}\right)$ can be seen as an operator which can be applied to both, harmonic sound fields and signals in different manners but always using delay-times, which are determined by $z_{\text {foc }}$, and $c_{\text {foc }}$. In Fig. 2 various simulated sound fields of the focused annular array are presented with the respective sound velocities of $c_{\text {med } 1}=1500 \mathrm{~m} / \mathrm{s}$ in medium 1 and $c_{\text {med2 }}=1600 \mathrm{~m} / \mathrm{s}$ in medium 2 . The focusing regime is calculated to focus at depths of $z=25 \mathrm{~mm}, 30 \mathrm{~mm}, 35 \mathrm{~mm}$, $40 \mathrm{~mm}$ and $45 \mathrm{~mm}$ using $c_{\mathrm{foc}}=1500 \mathrm{~m} / \mathrm{s}$.

The focussing regimes enables us to move the focus accurately along the acoustic axis, as can be seen by comparison of the sound fields within either horizontal row of Figure 2 In the upper row with $c_{\text {foc }}=c_{\text {med } 1}$, the focus appears at the aimed focus position. Focussing is possible in a range of $30 \mathrm{~mm}$ to $55 \mathrm{~mm}$. However, the focussing on $25 \mathrm{~mm}$ is not possible, as is generally expected because the near-field length of the single element restricts the lower limit of the focussing range. With focus location deeper inside the medium, the actual sensitivity zone is enlarged. The enlarged focus area predicts a decreased lateral resolution for regions with an increased distance to the transducer. Secondary maxima occur between the transducer and the focus, caused by the sparse design of the array. These secondary structures have also been detected in measurements [40].
In the lower row of Figure 2, we apply the same focusing regime as in row 1 . That means only the parameters of the propagation medium change while transducer parameters stay constant. The focus in row 2 with $c_{\text {foc }} \neq c_{\text {med2 }}$ appears closer to the transducer, because with higher SoS a virtual curvature with a smaller radius is generated. The effects of this incorrectly chosen focussing regime is best visible with the focusing $F(30 \mathrm{~mm}, 1500 \mathrm{~m} / \mathrm{s})$. Even though the effect appears minimal, we will show subsequently that we are able to use it for the novel approach to speed maps.

\section{Measurement approach}

The previous examinations show, that the amplitude of a reflected signal becomes maximal if both sound velocity and depth is chosen correctly. By independently iterating $c_{\text {foc }}$ and $z_{\text {foc }}$, one could determine the distance to a reflector as well as the average $S o S$ of the medium along the propagation path. However, such an approach would cause two issues: first, iterating over a 2D parameter space would be time consuming, and second and more crucial, strong, local maxima beside the global one could impact the evaluation. To reduce the dimension of the problem, the relationship between $S o S, T o F$ and path length is used. Because $z_{\mathrm{foc}}=c_{\mathrm{foc}} T o F / 2$, the focusing regime can be written as function dependent on the used sound velocity $c_{f o k}: F_{T o F}\left(c_{\text {foc }} \mid t_{T o F}\right)=F\left(c_{\text {foc }} t_{T o F} / 2, c f o c\right)$.

For a point reflector located on the acoustic axis at a depth of $z=35 \mathrm{~mm}$ in a medium with $c_{\text {med }}=1500 \mathrm{~m} / \mathrm{s}$, a reflected signal reaches the receiver with a time of flight $T o F=$ $46.7 \mu \mathrm{s}$. If we use this focus regime for a medium with a unknown SoS $c_{\text {med }}$, we could also say that we focus on a reflector that gives us an echo signal with $T o F=46.7 \mu \mathrm{s}$. For $c_{\mathrm{foc}}=1300 \mathrm{~m} / \mathrm{s}$, the focus regime for a reflector 'at $46.7 \mu \mathrm{s}$ ' yields $F(1300 \mathrm{~m} / \mathrm{s} \mid 46.7 \mu \mathrm{s})=F(30.4 \mathrm{~mm}, 1300 \mathrm{~m} / \mathrm{s})$. Accordingly, the focus regimes for $c_{\mathrm{foc}}=1400 \mathrm{~m} / \mathrm{s}, 1600 \mathrm{~m} / \mathrm{s}$ and $1700 \mathrm{~m} / \mathrm{s}$ are $F(1400 \mathrm{~m} / \mathrm{s} \mid 46.7 \mu \mathrm{s})=F(32.7 \mathrm{~mm}$, $1400 \mathrm{~m} / \mathrm{s}), F(1600 \mathrm{~m} / \mathrm{s} \mid 46.7 \mu \mathrm{s})=F(37.4 \mathrm{~mm}, 1600 \mathrm{~m} / \mathrm{s})$ and $F(1700 \mathrm{~m} / \mathrm{s} \mid 46.7 \mu \mathrm{s})=F(39.7 \mathrm{~mm}, 1700 \mathrm{~m} / \mathrm{s})$, respectively. For clarity, the focus regimes are given for approximate values in Figure 3 As can be seen, a local maximum at $z=30 \mathrm{~mm}$ appears only for $F(1500 \mathrm{~m} / \mathrm{s} \mid 46.7 \mu \mathrm{s})=$ $F(35 \mathrm{~mm}, 1500 \mathrm{~m} / \mathrm{s})$, i.e. the case for which $c_{\mathrm{foc}}=c_{\text {med }}$. In comparison, the resulting signal for all other focus regimes returns a much smaller value for $T o F=46.7 \mu$ s (shown as black lines in the sound fields). Gradually varying $c_{\text {foc }}$ results in the curve shown at the bottom of Figure 3 . As expected, the maximum is located exactly at the at the position $c_{\mathrm{foc}}=c_{\mathrm{med}}$.

\section{EXPERIMENTAL REALIZATION}

\section{A. Set-up}

In order to produce replicable result, a polyacrylamide phantom was developed that closely resemble the acoustic and thermal properties of tissue [41]. Its elastic properties can be adjusted by the ratio of acrylamide and bisacrylamide and the concentration of water. The phantom phantom consists of $26.9 \%$ acrylamide, $1.4 \%$ bisacrylamide, $71.7 \%$ water as well as ammonium persulphate and 


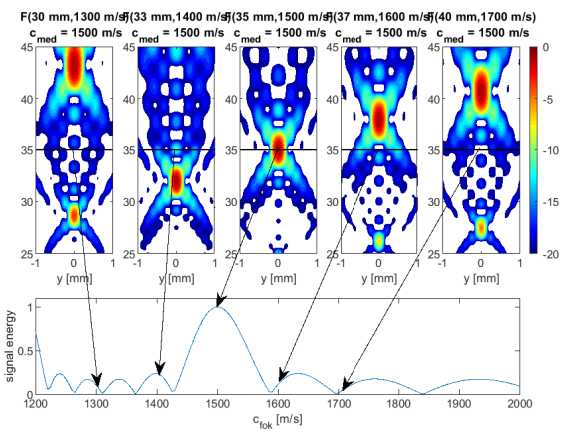

Fig. 3. Top: Resulting sound fields for focussing with various sound velocities and corresponding focus distances related by $t_{T o F}$ to a assumed reflector at $z=35 \mathrm{~mm}$ in a medium with $c_{\text {med }}=1500 \mathrm{~m} / \mathrm{s}$, The black lines mark the reflekor position and illustrates how much signal energy would be reflected from this depth. Bottom: Signal energy as a function of focusing sound velocity $c_{f o k}$. Data points, resulting from the example sound fields above are marked by arrows.

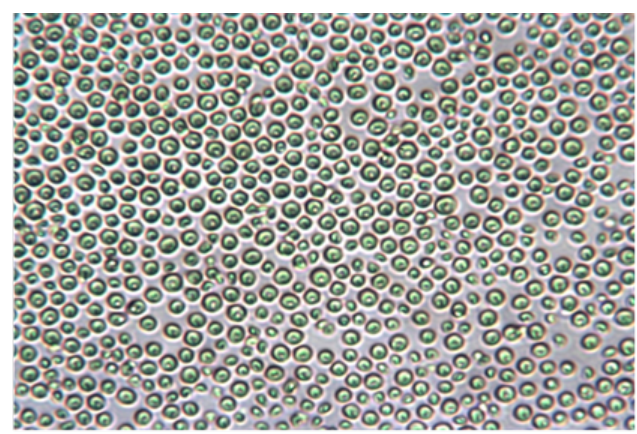

Fig. 4. Image of the gel with algea taken with an optical microscope.

$N, N, N^{\prime}, N^{\prime}$ - Tetramethylethylendiamine to start polymerisation. The mixture has a low attenuation but also a very low number of scattering particles. Therefore, the robust singlecelled green algae Chlorella Vulgaris, which can be easily cultivated, were added for scattering. The single alga has a diameter of $4 \mu \mathrm{m}$ to $10 \mu \mathrm{m}$ and the algae disperse regularly within the phantom, as can be seen in images taken with an optical microscope (Figure 4). As the algae are much smaller than the wavelength, we assume that the observed echoes are not caused by single algae but rather by algal clusters. Therefore, irregular signal shapes and various scattering characteristics can be expected on a small scale.

The phantom can be used in a variety of measurement setups: e.g. in a temperature-regulated water bath for measurements with constant sound velocities, or in a setup for local heating, as it is shown in Figure 5. To realize the necessary temperature gradient along the axis of the cylindrical phantom, adjustable heating transistors are placed on the top, while the bottom sits in a temperature regulating water bath, both connected to the phantom with metal plates.

\section{B. Data aquisition}

Ultrasonic measurements are performed with an annular array custom made by SONAXIS S.A, Besançon, france, with six annuli, a center frequency of $10 \mathrm{MHz}, 100 \%$ Bandwidth

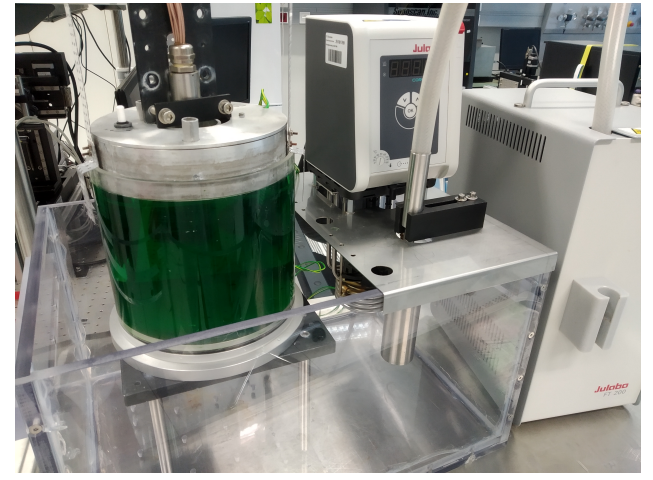

Fig. 5. Experimantal setup to generate a temperature gradient. For better visibility the lower water bath is not filled with water.

and a operating temperature up to $85^{\circ} \mathrm{C}$. It is pre-focussed with a curvature of $50 \mathrm{~mm}$. The dimensions of the annuli are given in Table 1: the inner and outer radii of each element $r_{\mathrm{i}}$ and $r_{\mathrm{a}}$, as well as the coordinates $r_{\mathrm{el}}$ and $z_{\mathrm{el}}$ used for focusing in Equation 1. How they are determined is discussed in section $\mathrm{V}$

TABLE I

GEOMETRY OF THE USED ANNULAR ARRAY.

\begin{tabular}{rrrrrrr}
\hline Element-No. & 1 & 2 & 3 & 4 & 5 & 6 \\
\hline$r_{i}[\mathrm{~mm}]$ & 0,5 & 5,05 & 7,13 & 8,72 & 10,07 & 11,25 \\
$r_{a}[\mathrm{~mm}]$ & 3,59 & 6,18 & 7,96 & 9,42 & 10,68 & 11,80 \\
$r_{\mathrm{el}}[\mathrm{mm}]$ & 2,56 & 5,64 & 7,56 & 9,08 & 10,38 & 11,53 \\
$z_{\mathrm{el}}[\mathrm{mm}]$ & 0,07 & 0,32 & 0,57 & 0,83 & 1,09 & 1,37 \\
\hline
\end{tabular}

The array is driven by a 16-channel pulser receiver unit (own development [42]), with a pulse length of $4 \mathrm{~ns}$ to $10 \mathrm{~ns}$, adjustable voltage ( $50 \mathrm{~V}$ to $400 \mathrm{~V}), 100 \mathrm{MHz}$ bandwidth and variable gain. Data acquisition is realized with two $\mathrm{GaGe}$ (Vitrek, LLC, Lockport, USA) Octupus digitizer boards (125 MS/s sample rate, 14-bit resolution). All data are collected so that a subsequent synthetic focusing is possible. That means that each element is driven independently and that the echo needs to be recorded with each element. The resulting signal for each element is digitized and stored separately. Afterwards the recorded signals are superposed, whereby the delay times between the signals can be chosen freely. Figure 6 shows an example of a signal obtained by emitting and receiving with the central element, averaged over 1000 signals to improve the signal-to-noise ratio. At the top, the whole signal is shown, including the surface echo from water to gel, at $t=42 \mu \mathrm{s}$, the backwall echo from gel to aluminum, at $t=105 \mu \mathrm{s}$, and a multiple reflection in the aluminum plate, at $t=$ $118 \mu \mathrm{s}$. Figure 6 (bottom) shows an enlarged section around the surface echo. Whereas there are no echoes before the surface echo, many echoes can be seen afterwards. These echoes are backscattered from the algae and show a variety of amplitudes and shapes. Hence the recorded signal supports the assumption that scattering is caused by algal clusters. Similar signals are obtained for all 36 combinations of emitters and receivers. 

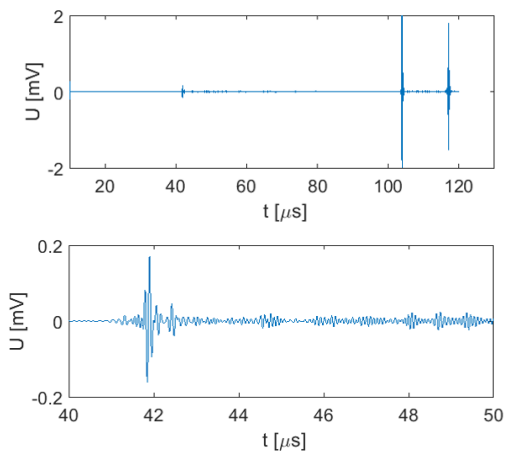

Fig. 6. Recorded signal by emitting and receiving with the central ring; top: whole echo including surface echo and back-wall echoes; bottom: signal part including surface echoes and backscattered echoes from the algae.

\section{Data evaluation}

As described above, we search the best fitted focus regime that produces a maximum in a single time window. In practice, we proceed as follows:

- selection of a time window of about $1 \mu$ s length and a position on the time axis with backscatter echoes from the algae.

- determination of the time of flight $t_{\mathrm{ToF}, \max }$ within the time window for which the signal, emitted and received with the center element, is at its maximal amplitude

- assumption of a (reasonable) SoS $c_{\text {foc }}$ and determination of the corresponding focus position $z_{\mathrm{foc}}=$ $c_{\text {foc }} t_{\text {ToF }, \max } / 2$

- synthetically focusing all signals with the parameters $z_{\text {foc }}$ and $c_{\text {foc }}$ and determination of resulting signal energy $E$ as well as the resulting signal amplitude $A$

- variation of $c_{\text {foc }}$ and calculation of signal energy $E\left(c_{\text {foc }}\right)$ and amplitude $A\left(c_{\text {foc }}\right)$

- determination of the average sound velocity by determining the maximum of one of the curves $c_{\text {med, } \mathrm{A}}=$ $\operatorname{argmax}\left(A\left(c_{\mathrm{foc}}\right)\right), c_{\text {med, } \mathrm{E}}=\operatorname{argmax}\left(E\left(c_{\mathrm{foc}}\right)\right)$

- checking for plausibility via comparing the maximal amplitude $A_{\max }$ with the average amplitude $A_{\mathrm{av}}$ and the requirement $A_{\max }>2 A_{\text {av }}$ (if only noise was evaluated, they would be nearly similar)

- shifting the time window stepwise along the recorded signal and repeating the previous steps in order to determine the average sound velocities $c_{\mathrm{av} ; i}$ at various observation points

- calculation of the local sound velocities $c_{\mathrm{loc} ; i}$

The width of the time window is determined by the demand that the frame should be much wider than the scatter echo length of about $0.4 \mu \mathrm{s}$ but not that large that several echoes occur within one window. Also the testing $\mathrm{SoS}\left(c_{\mathrm{foc}}\right)$ should be in a reasonable range. As the SoS of tissue is in a range of $1500 \mathrm{~m} / \mathrm{s}$ to $1700 \mathrm{~m} / \mathrm{s}$, values below $1300 \mathrm{~m} / \mathrm{s}$ or above $2000 \mathrm{~m} / \mathrm{s}$ are omitted, otherwise additional local maxima could occur, as discussed in section V (e.g. see Figure 12).

Figure 7 shows an example of a resulting curve of determined signal energy as a function of $c_{\mathrm{foc}}$ obtained from a measurement (blue) and from simulation (red). There is

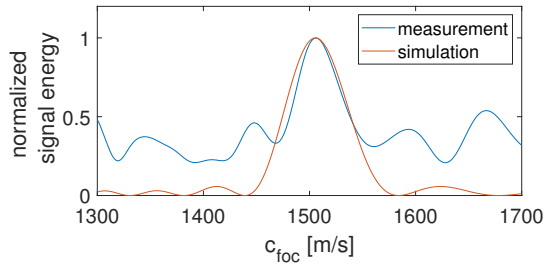

Fig. 7. Measured(blue) and simulated(red) signal energy as a function of the sound velocity $c_{\text {foc }}$.

a good match regarding peek position and peek width. But apart from the peak a much higher signal energy occurs in measurement. The reasons will be discussed in section V The figure also illustrates the criteria of the plausibility check. If the resulting energy is nearly constant and no significant maximum occurs, there are not sufficient scatterers within the window. In this case, the respective time window does not provide a meaningful SoS and is therefore disregarded. The figure also illustrates the necessity of chosing a sufficiant wide range of $c_{\text {foc }}$. If $c_{\text {foc }}$ is varied only in a range of the width of the main peak, e.g. $50 \mathrm{~m} / \mathrm{s}$, there won't be a significant difference between $A_{\max }$ and $A_{a v}$ and no check for plausibility is possible. After determining the correct average sound velocities $c_{\mathrm{av} ; i}$ for each frame position, the position $z_{i}$ of the scattering algae can be determined. The value of $c_{\mathrm{av} ; i}$ is the average sound velocity from the transducer to the corresponding focus depth. To determine the local sound velocity $c_{\text {loc; } i}$, Equation 5 can be used:

$$
c_{\mathrm{loc}, i}=\frac{c_{\mathrm{av}, i} z_{i}-c_{\mathrm{av}, i-1} z_{i-1}}{z_{i}-z_{i-1}}
$$

Of course, no local sound velocity can be determined for the first measurement at minimal focus distance. In general, we have to assume $c_{l o c ; 1}=c_{a v ; 1}$. But this does not affect the next measurement positions.

\section{Measurement Results}

In order to determine the accuracy of the approach, initial measurements were performed in a water bath to guarantee a constant temperature of $10{ }^{\circ} \mathrm{C}$ inside the phantom. As an example for the measurement at a temperature of $10^{\circ} \mathrm{C}$, Figure 8 shows the determined sound velocity as a function of ToF of the evaluated echo part. Subsequently, the determined sound velocity and the $T o F$ are used to calculate the reflector positions. Figure 9 shows the sound velocity as a function of measurement depth. Since the temperature and thus velocity are constant throughout the phantom, the observed deviations can be interpreted as the measurement uncertainty. The uncertainty is about $\pm 15 \mathrm{~m} / \mathrm{s}$ or $2 \%$. The determined sound velocity seems to decrease with increasing depth. This effect is thought to be due to inaccuracies of the attaching points used for focusing and has to be examined systematically in future work.

For comparison, the dependence of the sound velocity from temperature was determined with conventional sound velocity measurements. For this purpose we produced a phantom with 


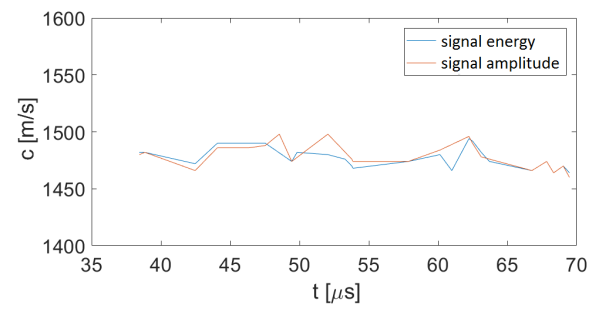

Fig. 8. Determined sound velocity as a function of time of flight.

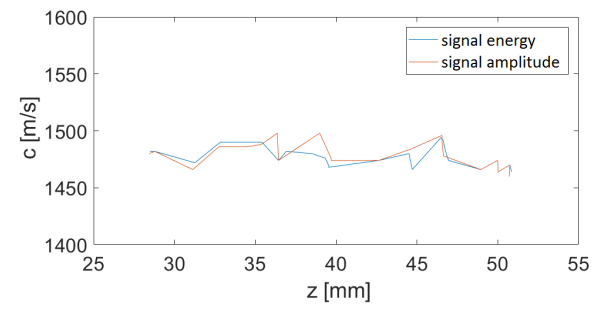

Fig. 9. Locally resolved sound velocity.

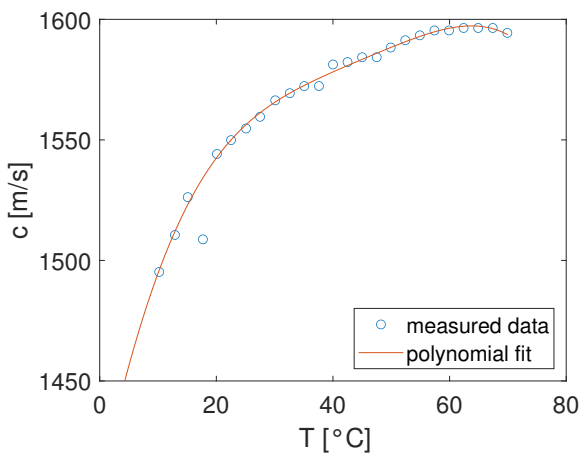

Fig. 10. Sound velocity as a function of temperature - conventional measurement.

two known reflectors, using the same gel but no algae. The reflectors were realized with a rod $(10 \mathrm{~mm}$ in height) on a plate. Aligning a transducer perpendicularly to the plate and centering over the rod, the echoes from the plate and from the top of the rod can be detected in one measurement without additional movement of the transducer. The $S o S$ inside the gel is determined from the difference of $T o F$ of the two reflectors. Heating the phantom gradually in water yields the measured curve of Figure 10 As the time of flight can be determined in this setup very precisely via correlation, we consider the main factors for the measurement uncertainty of about $0.5 \%$ in the manufacturing tolerances of the sample und the positioning tolerances of the scanner system. The curve in Figure 10 can be used to convert sound velocity maps into temperature maps.

The setup shown in Figure 5 was used to realize a temperature gradient. Only the bottom of the phantom sits in a water bath maintaining a constant temperature of $4{ }^{\circ} \mathrm{C}$. At the start of the measurement series at the time $t=0$ the heat source was turned on. Measurements were performed every $20 \mathrm{~s}$. For each measurement, the sound velocity distribution was determined as a function of time of flight (as in Figure 8), subsequently color-coded and depicted in Figure 11 along the y-axis. Each

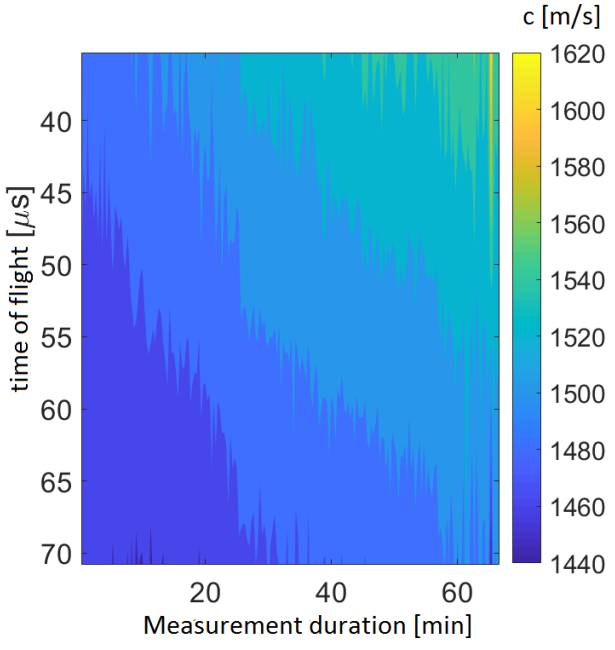

Fig. 11. Observation of the generation of a temperature gradient inside the PAA-gel-phantom, the time of flight (y-axis) corresponds to the measurement depth.

column in this figure represents a single measurement. The $\mathrm{x}$-axis shows the the duration of the measurement series. With increasing time, one can observe the development of the sound velocity gradient. The slight gradient at the beginning of the measurement series is due to the temperature difference between the water bath at the bottom and room temperature at the top of the phantom.

\section{THEORETICAL VALIDATION AND DETERMINATION OF ACCURACY}

The complexity of the evaluation makes it difficult to determine the measurement uncertainty by conventional methods like error propagation law. Therefore, the uncertainty is determined by the shape of the peak in Figure 7. If the assumptions of $z_{e l, i}$ and $r_{e l, i}$ for focusing are correct and $f_{\text {foc }}$ matches the $c_{\text {med }}$, the pressure at the position of the scatterer reaches its maximum and the peak appears at the correct position. The transducer properties affect the width of the peak and the electric properties of the measuring system, resulting in the signal to noise ratio (SNR), determine the accuracy of evaluating its position. For the purpose of evaluation, it is assumed that the scatterers are located only on the acoustic axis of the transducer and that they behave like point scatterers. As the simulated and the measured curve in Figure 7 coincide for the maximum value, sound-field calculations can be used to model the measurement process. In Figure 12, the calculated signal amplitude is depicted as a function $c_{\text {foc }}$ and of the reflector position $z_{\text {med }}$. Due to a signal to noise ratio of about $40 \mathrm{~dB}$ of the measuring system, values of and above $99 \%$ of the maximum cannot be differentiated. These boundaries are marked as red lines in Figure 12, Note that the measurement uncertainty increases with the measurement depth. This dependency of uncertainty from measurement depth is plotted in Figure 13. With 2\% and increasing from 1\% to $2 \%$ respectively, the experimentally determined uncertainties and the calculated ones fall in a convincingly similar range (cf. section IV. 


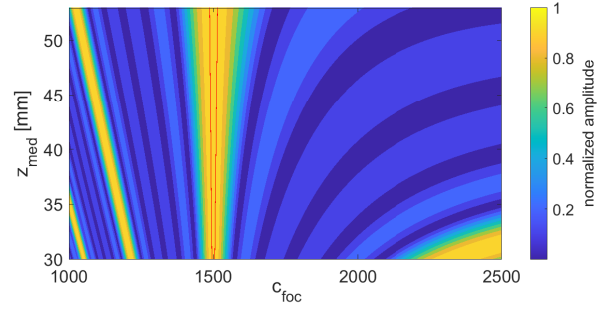

Fig. 12. calculated normalized signal energy as a function of testing sound velocity $c_{\text {foc }}$ and reflector position $z_{\text {med }}$-red lines added to indicate the SNR of $40 \mathrm{~dB}$

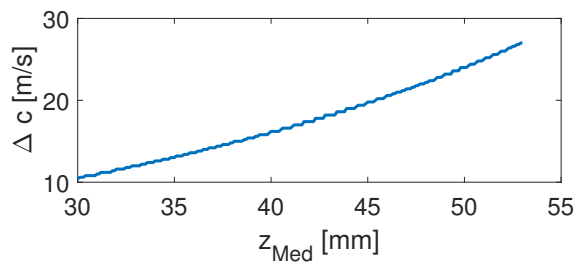

Fig. 13. Measurement uncertainty as a function of measurement depth.

Sound-field calculation can also be used to determine the lateral resolution. For this purpose, point reflectors are placed off the acoustic axis. In Figure 14, three calculated signal energy curves for off-axis scatterers are shown in comparison with the calculated on-axis curve. For an offset of $0.1 \mathrm{~mm}$, the peak of the curve is slightly diminished but maintains the same position of the maximum as in the on-axis curve. However, for an offset of $0.2 \mathrm{~mm}$ the peak already disappears and the resulting value would be rejected by the plausibility check.

That means that the proposed approach only evaluates signals from scatterers in very close proximity to the acoustic axis and and that the lateral resolution is better than $0.4 \mathrm{~mm}$. The modeling for off-axis scatterers also provides an explanation for the more prominent secondary maxima in the measured energy curve compared to the calculated one (as seen in Figure 7). Figure 14 shows that a scatterer with an offset of $0.3 \mathrm{~mm}$ produces a curve with two maxima on the left and the right of the maximum position of the on-axis curve. This kind of offset maxima increases the secondary structures if onaxis and off-axis scatterers are simultaneously present, as it is usually the case in tissue. This is due to the superimposition of the signals from on-axis and off-axis scatterers. For extreme cases, this superimposition of the on-axis and off-axis signals can cause a misleading peak at $c_{\text {foc }} \neq c_{\text {med }}$.

There are many further aspects, which can be taken into account. The measurement of the time of flight for each signal part is difficult, as it is best determined by correlation. But that is not possible due to the change in spectral composition (see Figure 6. Instead $t_{T o F}$ is determined by the maximum of the envelope. The reason for the different signal shapes is that the scattering is not caused by a single alga but by a cluster of them. One further important parameter is the calculation of the delay times $t_{n}$ for focusing (Equation 1). As the elements have a large area, the times are mainly affected by the chosen attaching points $\left(r_{e l}, z_{e l}\right)$. Now they are calculated so, that the

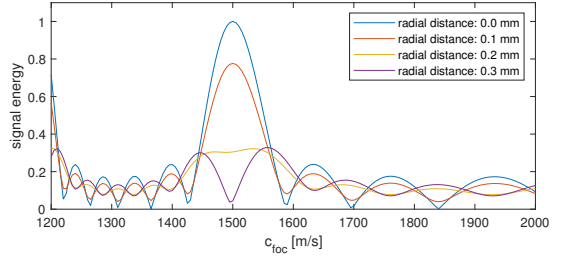

Fig. 14. Calculated signal amplitude for reflectors with an axial displacement. Squaring the curve leads to the signal energy curve depicted in Figure 7

ring element is split into two parts of same area.

$$
r_{e l}=\sqrt{\frac{r_{i}^{2}+r_{a}^{2}}{2}}
$$

Moving $r_{e l}$ in any direction yields to a systematic deviation in the determined sound velocity for the simulated data. If there is a systematic deviation in measurements this implies, that the transducer model does not fit its real properties. A more detailed examination of all factors affecting the accuracy of this measuring technique will be part of future work.

\section{CONCLUSION}

The proposed measurement technique allows to measure speed of sound locally and temporarily resolved. In comparison to state-of-the-art echo shift techniques, which can only measure sound velocity changes, the new approach provides absolute measures of sound velocity. Knowing the relationship between temperature and sound velocity, the new technique allows the locally accurate mapping of temperatures. Moreover, the technique works independently of any thermally caused tissue expansion.

In order to determine the reliability and accuracy of the newly established method, the method was successfully evaluated on tissue phantoms. With the used setup and with a window size of $1 \mu \mathrm{s}$, the local sound velocity can be determined with an uncertainty of $2 \%$. That means, the local uncertainty is also in the range of $2 \%$, while the accuracy of ToF is much lower. The achievable local resolution is about $1 \mathrm{~mm}$ in axial direction and about $0.4 \mathrm{~mm}$ in lateral direction. Mainly the used measurement hardware, especially the speed and memory capacity of the ADC board, limit the temporal resolution. For measurements up to a duration of a few minutes, it is easily possible to measure the SoS every second. Overall, the proposed method is the first ultrasonic method that fulfills the requirements for monitoring ablative methods, as formulated in [10]. Due to its ability of localized imaging and its potent local and temporal resolution, the new method meets the performance requirements for further medical applications. It is predestined for sound-velocity and temperature maps in tissue, for the improvement of beamforming, and for the correction of conventional B-scans.

Additionally, it was shown that the measurement process can be modeled effectively by sound-field simulations. The modeling allows to predict the dependency between the parameters (of array and tissue) and the measurement uncertainty. Therefore, sound-field simulations are a powerful tool to efficiently determine the ideal evaluation algorithm and to 
design optimized arrays for a variety of requirements and applications. The ability to determine the accuracy in advance is crucial for the efficiency of the design process because a conventional development process would be far too complicated due to the complexity and the number of parameters involved.

Future work will include the adaptation of the algorithm for arrays with line elements in order to enable 2D measurements and the application for medical monitoring and imaging.

\section{ACKNOWLEDGMENT}

The authors would like to thank the Deutsche Forschungsgemeinschaft (DFG) for financial support in project 260366138.

\section{REFERENCES}

[1] Ralf Seip and Emad S Ebbini. "Noninvasive estimation of tissue temperature response to heating fields using diagnostic ultrasound". In: IEEE Transactions on Biomedical Engineering 42.8 (1995), pp. 828-839.

[2] Roberto Maass-Moreno and Christakis A Damianou. "Noninvasive temperature estimation in tissue via ultrasound echo-shifts. Part I. Analytical model". In: The Journal of the Acoustical Society of America 100.4 (1996), pp. 2514-2521.

[3] S Srinivasan, Rafaella Righetti, and Jonathan Ophir. "Trade-offs between the axial resolution and the signalto-noise ratio in elastography". In: Ultrasound in medicine \& biology 29.6 (2003), pp. 847-866.

[4] Claudio Simon, Philip VanBaren, and Emad S Ebbini. "Two-dimensional temperature estimation using diagnostic ultrasound". In: IEEE transactions on ultrasonics, ferroelectrics, and frequency control 45.4 (1998), pp. 1088-1099.

[5] Jignesh Shah et al. "Ultrasound imaging to monitor photothermal therapy-Feasibility study". In: Optics Express 16.6 (2008), pp. 3776-3785.

[6] Dalong Liu and Emad S Ebbini. "Real-time 2-D temperature imaging using ultrasound". In: IEEE Transactions on Biomedical Engineering 57.1 (2009), pp. 12-16.

[7] Bastien Arnal, Mathieu Pernot, and Mickael Tanter. "Monitoring of thermal therapy based on shear modulus changes: II. Shear wave imaging of thermal lesions". In: IEEE transactions on ultrasonics, ferroelectrics, and frequency control 58.8 (2011), pp. 1603-1611.

[8] Rémi Souchon et al. "Monitoring the formation of thermal lesions with heat-induced echo-strain imaging: A feasibility study". In: Ultrasound in medicine \& biology 31.2 (2005), pp. 251-259.

[9] Josquin Foiret and Katherine W Ferrara. "Spatial and temporal control of hyperthermia using real time ultrasonic thermal strain imaging with motion compensation, phantom study". In: PLoS One 10.8 (2015), e0134938.

[10] Matthew A Lewis, Robert M Staruch, and Rajiv Chopra. "Thermometry and ablation monitoring with ultrasound". In: International Journal of Hyperthermia 31.2 (2015), pp. 163-181.
[11] Emiliano Schena et al. "Fiber optic sensors for temperature monitoring during thermal treatments: an overview". In: Sensors 16.7 (2016), p. 1144.

[12] Yufeng Zhou. "Noninvasive thermometry in highintensity focused ultrasound ablation". In: Ultrasound quarterly 33.4 (2017), pp. 253-260.

[13] Naomi R Miller, Jeffrey C Bamber, and Paul M Meaney. "Fundamental limitations of noninvasive temperature imaging by means of ultrasound echo strain estimation". In: Ultrasound in medicine \& biology 28.10 (2002), pp. 1319-1333.

[14] Bastien Arnal, Mathieu Pernot, and Mickael Tanter. "Monitoring of thermal therapy based on shear modulus changes: I. shear wave thermometry". In: IEEE transactions on ultrasonics, ferroelectrics, and frequency control 58.2 (2011), pp. 369-378.

[15] Nicolás Benech and Carlos A Negreira. "Monitoring heat-induced changes in soft tissues with 1D transient elastography". In: Physics in Medicine \& Biology 55.6 (2010), p. 1753.

[16] J-L Gennisson et al. "Ultrasound elastography: principles and techniques". In: Diagnostic and interventional imaging 94.5 (2013), pp. 487-495.

[17] F Piscaglia et al. "Ultrasound shear wave elastography for liver disease. A critical appraisal of the many actors on the stage". In: Ultraschall in der Medizin-European Journal of Ultrasound 37.01 (2016), pp. 1-5.

[18] Christoph F Dietrich et al. "EFSUMB guidelines and recommendations on the clinical use of liver ultrasound elastography, update 2017 (long version)". In: Ultraschall in der Medizin-European Journal of Ultrasound 38.04 (2017), e16-e47.

[19] Camelia Gianina Foncea et al. "Comparative study between pSWE and 2D-SWE techniques integrated in the same ultrasound machine, with Transient Elastography as the reference method". In: Medical ultrasonography 22.1 (2020), pp. 13-19.

[20] Nicolas Benech et al. "1-D elasticity assessment in soft solids from shear wave correlation: The timereversal approach". In: IEEE transactions on ultrasonics, ferroelectrics, and frequency control 56.11 (2009), pp. 2400-2410.

[21] Etana C Elegbe and Stephen A McAleavey. "Single tracking location methods suppress speckle noise in shear wave velocity estimation". In: Ultrasonic imaging 35.2 (2013), pp. 109-125.

[22] Stephen A McAleavey, Laurentius O Osapoetra, and Jonathan Langdon. "Shear wave arrival time estimates correlate with local speckle pattern". In: IEEE transactions on ultrasonics, ferroelectrics, and frequency control 62.12 (2015), pp. 2054-2067.

[23] Peter J Hollender et al. "Single-and multiple-tracklocation shear wave and acoustic radiation force impulse imaging: matched comparison of contrast, contrast-tonoise ratio and resolution". In: Ultrasound in medicine \& biology 41.4 (2015), pp. 1043-1057.

[24] Yufeng Deng et al. "Ultrasonic shear wave elasticity imaging sequencing and data processing using a ve- 
rasonics research scanner". In: IEEE transactions on ultrasonics, ferroelectrics, and frequency control 64.1 (2016), pp. 164-176.

[25] Thomas Ersepke, Tim C Kranemann, and Georg Schmitz. "2-d bayesian displacement estimation improves contrast-to-noise/resolution trade-off in shear wave elasticity imaging". In: 2019 IEEE International Ultrasonics Symposium (IUS). IEEE. 2019, pp. 13791382.

[26] Sergio J Sanabria et al. "Spatial domain reconstruction for imaging speed-of-sound with pulse-echo ultrasound: simulation and in vivo study". In: Physics in Medicine \& Biology 63.21 (2018), p. 215015.

[27] Moein Mozaffarzadeh et al. "Phase aberration correction in transcranial ultrasound imaging using averaged sound velocity map in delay-and-sum beamformer". In: 2019 IEEE International Ultrasonics Symposium (IUS). IEEE. 2019, pp. 1894-1897.

[28] Hanna Bendjador, Thomas Deffieux, and Mickaël Tanter. "The SVD beamformer: Physical principles and application to ultrafast adaptive ultrasound". In: IEEE transactions on medical imaging 39.10 (2020), pp. 3100-3112.

[29] Marion Imbault et al. "Robust sound speed estimation for ultrasound-based hepatic steatosis assessment". In: Physics in Medicine \& Biology 62.9 (2017), p. 3582.

[30] Marko Jakovljevic et al. "Local speed of sound estimation in tissue using pulse-echo ultrasound: Model-based approach". In: The Journal of the Acoustical Society of America 144.1 (2018), pp. 254-266.

[31] Hideyuki Hasegawa. "Recent Developments in Adaptive Beamforming". In: 2019 IEEE International Ultrasonics Symposium (IUS). IEEE. 2019, pp. 1063-1066.

[32] Patrick Stähli, Martin Frenz, and Michael Jaeger. "Reflection-mode speed-of-sound imaging using softprior limits". In: 2019 IEEE International Ultrasonics Symposium (IUS). IEEE. 2019, pp. 948-950.

[33] Richard Rau et al. "Ultrasound aberration correction based on local speed-of-sound map estimation". In: 2019 IEEE International Ultrasonics Symposium (IUS). IEEE. 2019, pp. 2003-2006.

[34] Mario Wolf and Elfgard Kühnicke. "Non-invasive and Locally Resolved Measurement of Sound Velocity by Ultrasound”. In: Sensors \& Transducers 184.1 (2015), p. 53.

[35] Mario Wolf et al. "Ultrasonic temperature monitoring in tissue phantoms by locally resolved measurement of longitudinal and transverse wave speed". In: Proceedings of Meetings on Acoustics ICU. Vol. 38. 1. Acoustical Society of America. 2019, p. 020004.

[36] GF Miller and H Pursey. "The field and radiation impedance of mechanical radiators on the free surface of a semi-infinite isotropic solid". In: Proc. R. Soc. Lond. A 223.1155 (1954), pp. 521-541.

[37] Elfgard Kühnicke. "Three-dimensional waves in layered media with nonparallel and curved interfaces: A theoretical approach". In: The Journal of the Acoustical Society of America 100.2 (1996), pp. 709-716.
[38] Elfgard Kühnicke. "Plane arrays-Fundamental investigations for correct steering by means of sound field calculations". In: Wave Motion 44.4 (2007), pp. 248261.

[39] Elfgard Kuehnicke and Uwe Voelz. "Transient wave field calculation for ultrasonic transducers using integral transform methods". In: Acoustical Imaging. Springer, 1997, pp. 343-348.

[40] Michael Lenz et al. "Transducer characterization by sound field measurements". In: IEEE transactions on ultrasonics, ferroelectrics, and frequency control 60.5 (2013), pp. 998-1009.

[41] Cyril Lafon et al. "Gel phantom for use in highintensity focused ultrasound dosimetry”. In: Ultrasound in medicine \& biology 31.10 (2005), pp. 1383-1389.

[42] Norbert Gust and Falk Schellhorn. "High frequency 16 channel ultrasonic microscope for annular arrays". In: 2012 IEEE International Ultrasonics Symposium. IEEE. 2012, pp. 1-3.

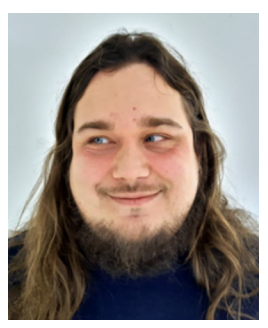

Mario Wolf was born in Leipzig, Germany, in 1985. He studied electrical engineering at the Technical University of dresden and was awarded a diploma degree in electrical engineering (specialization in technical acoustics) in 2011. Since 2011, he has been with the solid-state electronics laboratory of the Technical University of Dresden, where he continues his research in the field of ultrasound measuring technology. His current research interests concentrate on the theory of sound propagation and sound field simulations and signal processing.

Emanuel Leipner was born on October 12, 1992 in Dresden, Germany. He graduated in electrical engineering with specialization in information technology and high frequency technology in 2020 from the Technical University of Dresden. Since Autumn 2020 he is a member of the working group for ultrasonic measurement techniques at the Institute for Solid State Electronics at the Dresden University of Technology. His research focus is on hardware development and signal processing. .

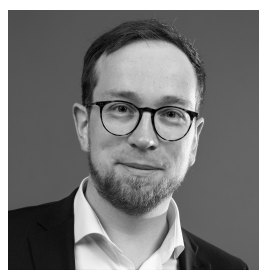

Christian Kupsch studied electrical engineering at TU Dresden with a focus on information technology and signal processing. His final thesis in the field of optical flow metrology was awarded by the Association for Electrical, Electric, and Information Technologies (VDE). He was a research assistant at the Laboratory of Measurement and Sensor System Technique at TU Dresden until early 2020. For his work on super-resolution flow imaging in suspensions, he received the RWB Stephens Prize at the 2019 International Congress on Ultrasonics (ICU) in Bruges, Belgium. In 2020, he received a Ph.D. degree in electrical engineering from TU Dresden, Germany and worked as a Postdoc at the Institute of Solid State Electronics, TU Dresden until early 2021.

Since april 2021, he has been a professor and head of the Measurement, Sensor and Embedded Systems Laboratory (MSE Lab) at TU Bergakademie Freiberg, Germany. . 


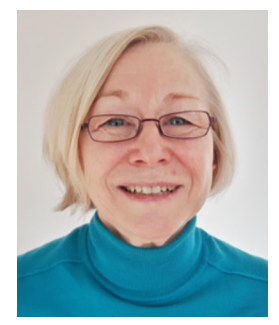

Elfgard Kühnicke was born on June 6, 1954, in Nordhausen, Germany. she received her diploma degree in physics from the University of Leipzig and her Ph.d. degree in physics from the Otto vonGuericke-University in Magdeburg in 1985. From 1978 to 1986 , she worked at the Technical University of Zittau in the field of acoustic emission, and from 1988 to 1991 at the academy of sciences of the GDR in the field of ultrasonics. In 1993, she joined the Institute of Technical Acoustics at the Technical University of Dresden, where she started working as a postdoctoral research fellow. after receiving her postdoctoral lecturer qualification (habilitation) in ultrasound technology in 2000, she continued working as a university lecturer and researcher. In 2008, she was awarded a professorship as an associate professor and became the head of the ultrasound work group at the solid-state electronics laboratory of the Technical University of Dresden. Her research interests include ultrasonic sensor design, the modeling of ultrasonic fields and acoustic emission processes, and the development of novel ultrasonic measuring techniques for medicine and NDT. 